\title{
Sis \\ Enhancement of Images Using Contrast Image Enhancement Techniques
}

\author{
S. R. Suralkar \& Seema Rajput \\ SSBTCOET, Bhambori, Dist. Jalgaon
}

\begin{abstract}
Fingerprints are the oldest and most widely used form of biometric identification. The performance of any fingerprint recognizer highly depends on the fingerprint image quality. Different types of noises in the fingerprint images pose greater difficulty for recognizers. However, fingerprint images are rarely of perfect quality. They may be degraded and corrupted due to variations in skin and impression conditions. Thus, image enhancement techniques are employed prior to minutiae extraction to obtain a more reliable estimation of minutiae locations. Most Automatic Fingerprint Identification Systems (AFIS) use some form of image enhancement. Therefore, this paper describes various techniques for fingerprint image enhancement
\end{abstract}

Keywords - Contrast enhancement, Histogram equalization, PSNR, Spatial Domain method

\section{INTRODUCTION}

Fingerprints have been used for over a century and are the most widely used form of biometric identification. Fingerprint identification is commonly employed in forensic science to support criminal investigations, and in biometric systems such as civilian and commercial identification devices. Despite this widespread use of fingerprints, there has been little statistical work done on the uniqueness of fingerprint minutiae.

The fingerprint of an individual is unique and remains unchanged over a lifetime. A fingerprint is formed from an impression of the pattern of ridges on a finger. A ridge is defined as a single curved segment, and a valley is the region between two adjacent ridges $[1,2]$. The minutiae, which are the local discontinuities in the ridge flow pattern, provide the features that are used for identification. Details such as the type, orientation, and location of minutiae are taken into account when performing minutiae extraction

A fingerprint pattern is characterized by a set of ridgelines that often flow in parallel, but intersect and terminate at some points. The uniqueness of a fingerprint is determined by the local ridge characteristics and their relationships [3]. Most automatic systems for fingerprint comparison are based on minutiae matching. Minutiae characteristics are local discontinuities in the fingerprint pattern and represent the two most prominent local ridge characteristics: terminations and bifurcations. A ridge termination is defined as the point where a ridge ends abruptly, while ridge bifurcation is defined as the point where a ridge forks or diverges into branch ridges (Figure 1). A typical fingerprint image contains about 40-100 minutiae [4].

The performance of minutiae extraction algorithms relies heavily on the quality of the fingerprint images. The ridge structures in poor-quality fingerprint images are not always well defined and, hence, cannot be correctly detected. This might result in the creation of spurious minutiae and the ignoring of genuine minutiae. Therefore, large errors in minutiae localization may be introduced. Examples of poor quality fingerprint images are shown in Figure Figure 2. In order to ensure robust performance of a minutiae extraction algorithm, an enhancement algorithm, which can improve the clarity of the ridge structures, is necessary.

\section{BIOMETRICS TECHNIQUES}

Biometrics techniques are divided into two categories i.e Physiological (fingerprints, face, iris, DNA, retina,voice, hand geometry, palm print, retinal scan etc.) and Behavioral (gait, signature etc). These physiological or behavioral Characteristics are used for human identification on the basis of their universality, uniqueness, permanence and collectability .These system merely used in various application and systems where the authentication and identification of human being required, like Defense, law, crime, banking,communication etc. Fingerprint recognition system is based on two basic premises. 1) Persistence: The basic characteristics of fingerprint do not change with time i.e.preserve its characteristics and shape form birth to death. 2)Individuality: The fingerprint is unique to an individual [5].

AFAS (Automatic Fingerprint Authentication System) 
Components of AFIS are: [6][7][8]

1. Physical Fingerprint required as input.2. Input is processed by using various image processing tools and databases and Classification of Fingerprints. The basic fundamental steps of these systems (see Figure 1)are image acquisition, pre-processing segmentation, enhancement etc), feature extraction, matching along with classification through databases. Authentication or verification systems authenticate the person's identity by comparing the own biometric template(s) stored in database (One-to-One comparison). An identification system recognize by an individual by searching the entire templates in database for match (One-to-Many Comparison) [9]

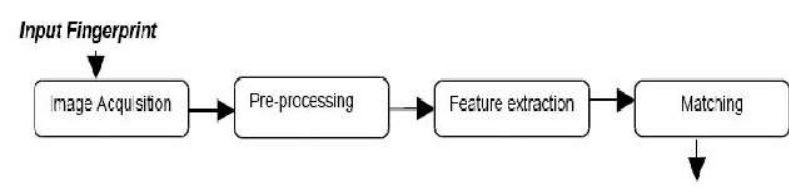

Decision for Authentication / Identification

Fig. 1 : Fundamental steps of Automatic Fingerprint Recognition System

\section{FINGERPRINT IMAGE PRE- PROCESSING}

The captured fingerprint image in RGB format is first converted to gray scale [0-255] followed by the normalization process.

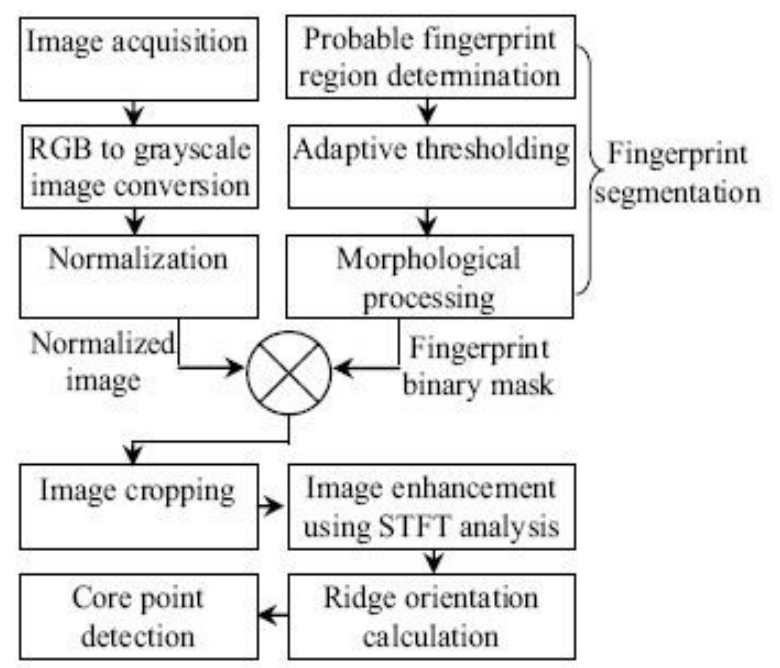

Fig. 2 : Flow Chart of the Fingerprint Preprocessing

Parallelly, we determine the probable fingerprint region. The adaptive thresholding followed by the morphological processing are performed on the determined probable fingerprint region in order to create the fingerprint binary mask. The normalized image is then multiplied with the fingerprint binary mask. The resulting image is cropped and enhanced by using the STFT based technique. Finally, the ridge orientation is calculated and the core point detection is done on the enhanced image. The process flow of the fingerprint preprocessing is shown in Figure 2

\section{IMAGE ENHANCEMENT}

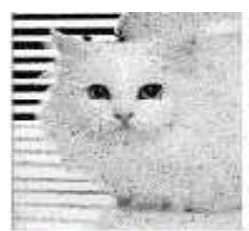

Distorted Image

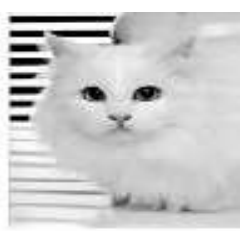

Enhanced Irrage
Fig. 3 : Showing the effect of Image Enhancement

Image enhancement is basically improving the interpretability or perception of information in images for human viewers and providing 'better' input for other automated image processing techniques(see Figure 3). The principal objective of image enhancement is to modify attributes of an image to make it more suitable for a given task and a specific observer. During this process, one or more attributes of the image are modified. The choice of attributes and the way they are modified are specific to a given task. Moreover, observer-specific factors, such as the human visual system and the observer's experience, will introduce a great deal of subjectivity into the choice of image enhancement methods. There exist many techniques that can enhance a digital image without spoiling it. The enhancement methods can broadly be divided in to the following two categories:

1. Spatial Domain Methods

2. Frequency Domain Methods

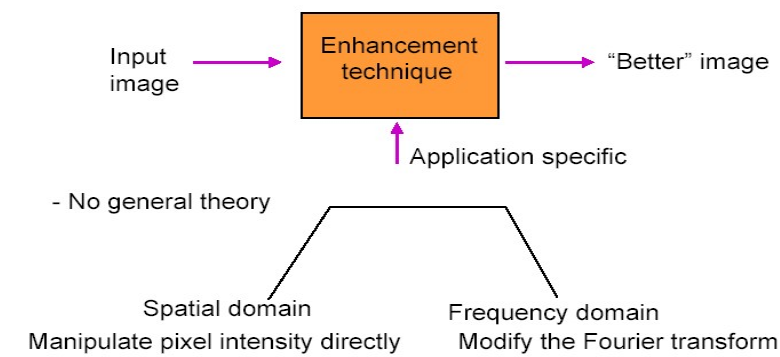

Fig. 4 : Image Enhancement in the Spatial Domain

In spatial domain techniques [10]we directly deal with the image pixels. The pixel values are manipulated to achieve desired enhancement. In frequency domain methods, the image is first transferred in to frequency domain. It means that, the Fourier Transform of the image is computed first. All the enhancement operations are performed on the Fourier transform of the image and then the Inverse Fourier transform is performed to get the resultant image. These enhancement operations are performed in order to modify the image brightness, contrast or the distribution of the grey levels. As a consequence the pixel value (intensities) of the output image will be modified according to the transformation function applied on the input values into image $g$ using $T$. (Where $T$ is the transformation. The values of pixels 
in images $f$ and $g$ are denoted by $r$ and $s$, respectively. As said, the pixel values $r$ and $s$ are related by the expression,

$$
s=T(r)
$$

Where $T$ is a transformation that maps a pixel value $r$ into a pixel value $s$. The results of this transformation are mapped into the grey scale range as we are dealing here only with grey scale digital images. So, the results are mapped back into the range $[0, \mathrm{~L}-1]$, where $\mathrm{L}=2 \mathrm{k}, \mathrm{k}$ being the number of bits in the image being considered. So, for instance, for an 8-bit image the range of pixel values will be $[0,255]$.

Many different, often elementary and heuristic methods[11] are used to improve images in some sense. The problem is, of course, not well defined, as there is no objective measure for image quality. Here, we discuss a few recipes that have shown to be useful both for the human observer and/or for machine recognition. These methods are very problem-oriented: a method that works fine in one case may be completely inadequate for another problem. In this paper basic image enhancement techniques have been discussed with their mathematical understanding. This paper will provide an overview of underlying concepts, along with algorithms commonly used for image enhancement.The paper focuses on spatial domain techniques for image enhancement .

\subsection{Image Enhancement Techniques}

\section{Spatial domain methods :}

The value of a pixel with coordinates $(x, y)$ in the enhanced image is the result of performing some operation on the pixels in the neighborhood of $(x, y)$ in the input image, $F$.Neighborhoods can be any shape, but usually they are rectangular. It Contains

- Negative Image Enhancement

- Histogram Image Enhancement

- Contrast Image Enhancement, etc

Negative Image Enhancement

The most basic and simple operation in digital image processing is to compute the negative of an image. The pixel gray values are inverted to compute the negative of an image. For example, if an image of size $\mathrm{R}$ $\mathrm{x} C$, where $\mathrm{R}$ represents number of rows and $\mathrm{C}$ represents number of columns, is represented by $\mathrm{I}(\mathrm{r}, \mathrm{c})$. The negative $\mathrm{N}(\mathrm{r}, \mathrm{c})$ of image $\mathrm{I}(\mathrm{r}, \mathrm{c})$ can be computed as

$$
\mathrm{N}(\mathrm{r}, \mathrm{c})=255-\mathrm{I}(\mathrm{r}, \mathrm{c})
$$

where $0<=\mathrm{r}<=\mathrm{R}$ and $0<=\mathrm{c}<=\mathrm{C}$.It can be seen that every pixel value from the original image is subtracted from the 255. The resultant image becomes negative of the original image. Negative images are useful for enhancing white or grey detail embedded in dark regions of an image.
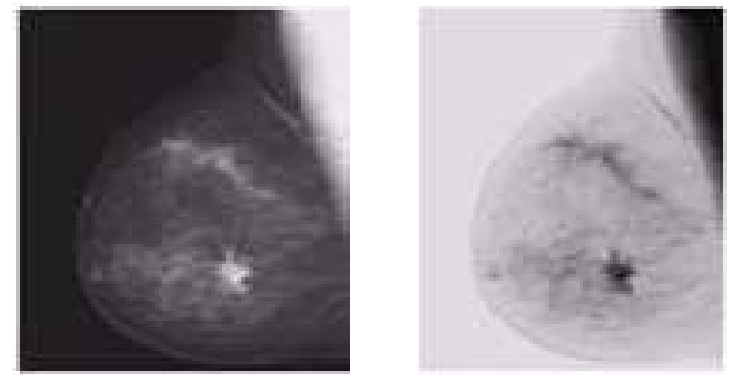

a) Original digital mammogram b)Negative image obtained by negative transformation

Fig. 5 : Note how much clearer the tissue is in the negative image of the mammogram

\section{Histogram Image Enhancement}

The histogram of a digital image with intensity levels in the range $[0, \mathrm{~L}-1]$ is a discrete function

$$
\mathrm{h}\left(\mathrm{r}_{\mathrm{k}}\right)=\mathrm{n}_{\mathrm{k}}
$$

where, $r_{k}$ is $k$ th intensity value and $n_{k}$ is number of pixel in the image with intensity $r_{k}$

$$
\mathrm{p}\left(\mathrm{r}_{\mathrm{k}}\right)=\mathrm{n}_{\mathrm{k}} / \mathrm{MN}, \quad \mathrm{k}=0,1, \mathrm{~L}-1
$$

Histograms are frequently normalized by the total number of pixels in the image. Assuming an $M \times N$ image, a normalized histogram,is related to probability of occurrence of $r k$ in the image

\section{RESULT AND DISCUSSIONS}

It is clearly seen that the Contrast Image enhancement method returns the best results when studied with all available database samples as compare with others as,indicated in below figures .Image 25_2.tif (Figure 6)shows the best results as compare with the others from FVC2004 Database no. 2 The parameters values obtained are Given in Table no 1

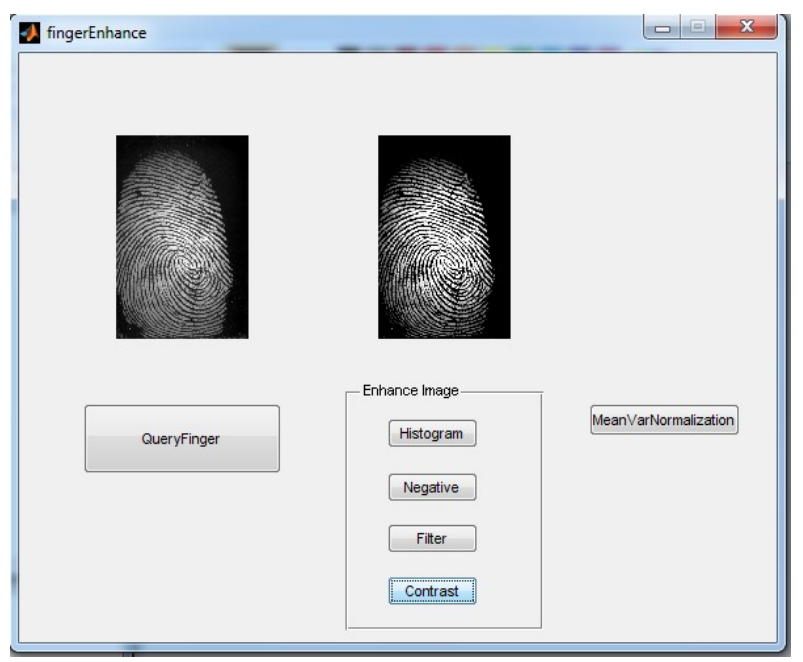

In this paper, we have studied various Image enhancement techniquessuch as Contrast ,negative ,etc. Using these techniques it is possible to detect the 
discontinuities and similarities occurred in Images and there quality in overall view of image.

In Contrast Image enhancement we have observed that as PSNR (Peak Signal to Noise Ratio) increases which yields the quality of the images and same time Normalized Error are proportionally increases.We have got the image 25 2.tif from the collection of $\mathrm{Db} 2$ having a better quality result as shown in Figure 6

In Histogram Image enhancement we have observed that as $\mathrm{N}$ ( Gray Scale value in number) increases, MSSIM

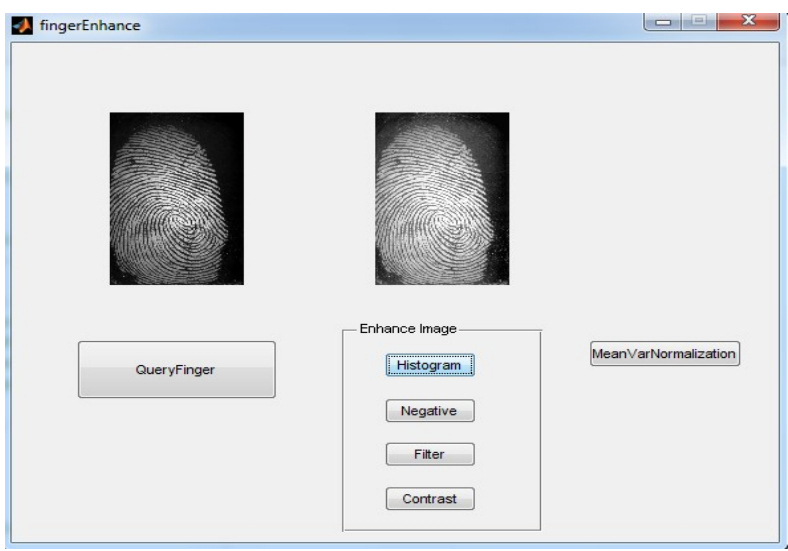

Fig. 7: Result of Histogram Image Enhancement

PSNR(Peak Signal to Noise Ratio) decreases and which yields the bad quality of the images and same time Normalized Error are proportionally decreases .We have got the image 61_6.tif from the collection of Db3 having a better quality result at $\mathrm{N}=100$ as shown in Figure 7

In Negative Image enhancement we have observed that as PSNR (Peak Signal to Noise Ratio) increases which yields the quality of the images and same time the Normalized Error are proportionally increases. We have got the image 11 2.tif from the collection of $\mathrm{Db} 4$ having a better quality result as shown in Figure 8

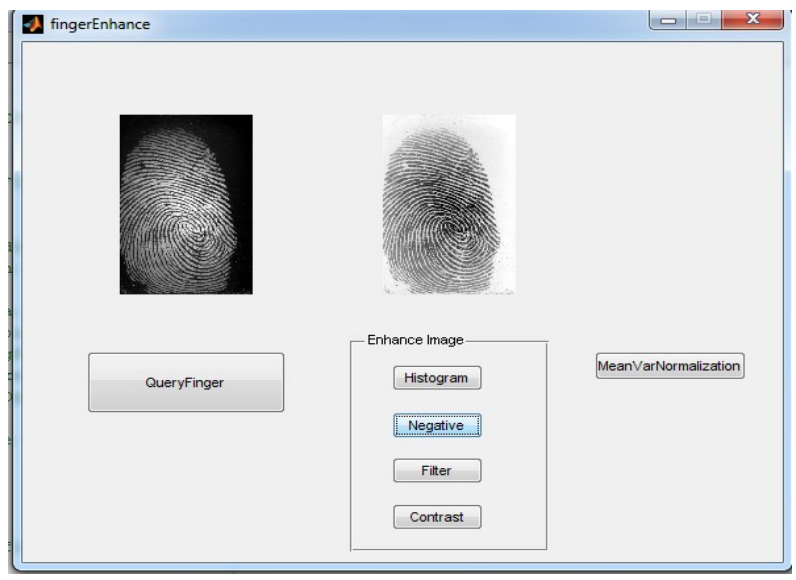

Fig. 8 : Result of Negative Image Enhancement
Out of these studied methods we are in the position to conclude that the contrast Image enhancement is the one of the best method within the studied techniques as shown in Table 1below.

\begin{tabular}{|l|l|l|l|}
\hline \multirow{2}{*}{ Parameter } & \multicolumn{3}{|l|}{ Enhancement Techniques } \\
\cline { 2 - 4 } & Histogram & Negative & Contrast \\
\hline PSNR & 24.0811 & 24.8234 & 33.0532 \\
\hline $\begin{array}{l}\text { Normalized } \\
\text { Error }\end{array}$ & 0 & 12.8883 & 17.2961 \\
\hline
\end{tabular}

Table 1: The contrast Image enhancement is the one of the best method within the studied techniques

\section{CONCLUSION}

Peak signal-to-noise ratio, often abbreviated PSNR, is the ratio between the maximum possible power of a signal and the power of corrupting noise that affects the fidelity of its representation. The PSNR is most commonly used as a measure of quality of reconstruction of lossy compression codecs (e.g., for image compression). The signal in this case is the original data, and the noise is the error introduced by compression higher PSNR would normally indicate that the reconstruction is of higher quality. It is clearly seen that the Contrast Image enhancement method returns the best results when studied with all available database samples as compare with others

\section{VII.REFERENCES}

[1] L. Hong, Y. Wan, and A. Jain. "Fingerprint image enhancement: Algorithm and performance evaluation," IEEE Transactions on Pattern Analysis and Machine Intelligence, 20(8):777789, August 1998.

[2] Y. He, J. Tang, X. Luo, and T. Zhang, "Image enhancement and minutiae matching in fingerprint verification," Pattern Recognition Letters, 24, 2003.

[3] Maltoni, D., Maio, D., Jain, A. K., and Prabhakar, S. Handbook of Fingerprint Recognition. Springer, 2003.

[4] Chaohong Wu, Zhixin Shi and Venu Govindaraju "Fingerprint Image Enhancement Method Using Directional Median Filter"

[5] Salil Prabhakar, "Fingerprint classification and matching using filterbank", Ph. D. Thesis, 2001

[6] Xia,X. and O'Gorman, L.: Innovations in fingerprint capturedevices. Journal of Pattern Recognition, Pergamon Press, Vol. 36, No. 2, pp. 361-370, 2002 
[7] Jain,A., Hong,L. and Bolle,R.: On-Line Fingerprint Verification. IEEE-PAMI, Vol.19, No.4,pp. 302-314, Apr.1997.

[8] Zhou Wang,Alan Conrad Bovik'sJain,A., “Image Quality Assessment :from error visibility to structure similarity IEEE transaction On image processing" Vol.13 No4 April2004]
[9] D. Maltoni, Dmaio, A.K. Jain, S. prabhakar, "Hand book of Fingerprint ecognition", springer, 2003

[10] Bhabatosh Chanda and Dwijest Dutta Majumder, 2002, Digital Image Processing and Analysis.

[11] R.W.Jr. Weeks,(1996). Fundamental of Electronic Image Processing . Bellingham: SPIE Press] 